\title{
Eosinophilic granulomatosis with polyangiitis (formerly Churg-Strauss syndrome): where are we now?
}

\author{
Matthieu Groh¹, Christian Pagnoux ${ }^{2}$ and Loïc Guillevin ${ }^{1}$
}

Affiliations: ${ }^{1}$ Dept of Internal Medicine, National Referral Center for Rare Autoimmune and Systemic Diseases (Vasculitis, Scleroderma), Hôpital Cochin, APHP, Université Paris Descartes, Paris, France. ${ }^{2}$ Division of Rheumatology, Dept of Medicine, Mount Sinai Hospital, University of Toronto, Toronto, ON, Canada.

Correspondence: Matthieu Groh, Dept of Internal Medicine, Hôpital Cochin, 27, rue du faubourg Saint-Jacques, 75679 Paris Cedex 14, France. E-mail: matthieu.grohdacch.aphp.fr

$\circ$ @ERSpublications

The EGPA Consensus Task Force's first recommendations for EGPA diagnosis and individualised patient management http://ow.ly/RZ3Sw

Antineutrophil-cytoplasm antibody (ANCA)-associated vasculitides (AAV), classified as small-sized vessel vasculitides, include: granulomatosis with polyangiitis (GPA) (formerly Wegener's granulomatosis disease), microscopic polyangiitis (MPA), and eosinophilic granulomatosis with polyangiitis (EGPA) (formerly Churg-Strauss syndrome) [1]. Although they share some common features, EGPA has certain specificities, namely asthma, blood and tissue eosinophilia, and frequent ear, nose and throat (ENT) involvement, while ANCA (targeting neutrophil myeloperoxidase) are found in only a subset of patients $(30-70 \%)[2,3]$. Moreover, EGPA diagnosis can be challenging, as its manifestations can overlap with those of primary hypereosinophilic syndromes (HES) [4].

Substantial progress has been made over the last decades in understanding AAV pathophysiology and treating those diseases. Before the advent of glucocorticoid and immunosuppressant therapy, survival was poor ( $\sim 20 \%$ at 2 years), but now reaches $\sim 80 \%$ at 5 years [5]. In 2009 , the European League Against Rheumatism (EULAR) published recommendations for managing small- and medium-sized vessel vasculitides [6]. However, because EGPA's prevalence (7-13 per 1000000 inhabitants) [7] is lower than that of other AAVs, with fewer trials having been conducted, most of those recommendations addressed only GPA and MPA. A recent issue of the European Journal of Internal Medicine published recommendations for EGPA evaluation and management devised by the EPGA Consensus Task Force [8]. The European Respiratory Society and Foundation for the Development of Internal Medicine in Europe commissioned the EGPA Consensus Task Force that included 23 vasculitis experts from different subspecialties from five European Union countries, the USA and Canada. Using a modified multistep Delphi process, a systematic literature review and GRADE-method-defined levels of evidence [9], 22 recommendations were established.

How should EGPA be diagnosed? First, the recommendations provide a detailed guide to the initial diagnosis work-up in the context of eosinophilia and asthma. Moreover, the definitions of disease remission and relapse now recognise that ENT manifestations and/or asthma flares may not necessarily reflect vasculitis activity and that these symptoms should be monitored separately.

How should EGPA be managed? Being a rare disease, EGPA often goes unrecognised. Because inappropriate therapeutic decisions can impact its prognosis [10], EGPA patients should ideally be managed in, or at least in collaboration with, centres with expertise in AAV care. Once the diagnosis is made, poor-prognosis manifestations, e.g. kidney, heart (the leading cause of EGPA-related deaths), central nervous system and gastrointestinal involvements should be sought [11]. Indeed, they require a remission-induction regimen

Received: June 182015 | Accepted: June 192015

Disclosures can be found alongside the online version of this article at erj.ersjournals.com

Copyright OERS 2015 
combining glucocorticoids (the cornerstone of EGPA therapy) and another immunosuppressant (e.g. oral cyclophosphamide or intravenous pulses). Life-threatening symptoms require initial methylprednisolone pulses. Immunosuppressants should also be prescribed to patients with severe alveolar haemorrhage, eye involvement (albeit rare in EGPA) and/or fulminant mononeuritis multiplex. However, glucocorticoids alone may suffice for patients without life- and/or organ-threatening disease manifestations; the randomised placebo-controlled CHUSPAN 2 trial (www.clinicaltrials.gov NCT00647166) is currently evaluating the potential benefit of adjunctive azathioprine as a glucocorticoid sparing agent. Once EGPA remission has been achieved, the relapse rate is high and many patients remain glucocorticoid dependent [12]. As with other AAVs, EGPA patients would probably benefit from maintenance therapy to avoid relapses and allow glucocorticoid tapering. Unlike for other AAVs [13-15], no controlled study has compared immunosuppressants for EGPA for that latter purpose. Therefore, azathioprine or methotrexate can be prescribed equally. The optimal duration of maintenance therapy remains unknown but the task force recommends at least $18-24$ months.

In addition to conventional immunosuppressants, immunotherapy (especially drugs with antagonist effect(s) against the T-helper cell (Th) 2 immune response) has also been tried in EGPA. Interferon- $\alpha$ was the first agent prospectively evaluated; although it obtained disease remission and allowed substantial lowering of the prednisone dose for some patients, numerous associated adverse events make it unacceptable as first-line treatment. Conversely, although anti-IgE therapy (omalizumab) efficacy in patients with high serum IgE titres was noted in several case reports [16], more information is needed before any recommendation can be formulated. Rituximab, a chimeric monoclonal antibody that targets B-lymphocytes, is now approved to induce GPA and MPA remissions [17], and has demonstrated effectiveness as maintenance [18]. Accumulating data on rituximab indicate that ANCA-positive EGPA patients seem to have higher remission rates than patients who are ANCA-negative [19]. To date, and although not licensed for EGPA, the task force suggests that using rituximab seems reasonable for ANCA-positive patients with renal involvement or severe refractory disease, for whom traditional cytotoxic agents are contraindicated or undesirable (e.g. to prevent cyclophosphamide-induced gonadal toxicity in younger patients and/or urinary bladder toxicity in those who have already received high cumulative cyclophosphamide doses). The French Vasculitis Study Group's prospective randomised REOVAS trial (rituximab induction therapy versus standard care) is about to begin and should help define rituximab's place in the therapeutic armamentarium for EGPA. Targeting interleukin (IL)-5 (the major eosinophil survival factor) also holds promise for EGPA. Mepolizumab, a humanised monoclonal antibody effective against eosinophilic asthma [20], has shown promising results in two pilot studies on EGPA [21, 22]. The pharmaceutical industry-driven and sponsored MIRRA study (www.clinicaltrials.gov NCT02020889) is currently evaluating it for EGPA with first results expected in late 2016.

What are the main challenges of EGPA management? Physicians are confronted with several specific and difficult-to-manage clinical situations. First, chronic asthma is a major burden for EGPA patients and asthma bouts can occur repeatedly throughout the disease course, especially when the prednisone dose is $<10 \mathrm{mg} \cdot \mathrm{day}^{-1}$ and/or the eosinophil count exceeds $500 \cdot \mathrm{mm}^{-3}$ [12]. EGPA probably encompasses a broad heterogeneity of endotypes that could benefit from different treatments. Although several biotherapies for asthma have been examined or are currently under investigation, so far only omalizumab and mepolizumab have been used for EGPA. Other anti-IL-5, IL-4 and/or IL-13 targeted agents warrant being evaluated for this vasculitis. Second, cardiac involvement is the leading cause of EGPA-associated deaths, at least in historical series, and is more frequent in ANCA-negative patients [2]. Thus, it should systematically be sought early and regularly thereafter. Prompt glucocorticoids and cyclophosphamide use may enable cardiac function recovery. Heart transplantation for EGPA-refractory patients is feasible and carries a fair prognosis [23]. Peripheral neuropathy is frequent and disabling. Recent findings suggest patients with symptomatic pain initially treated with glucocorticoids alone are at higher risk of requiring treatment intensification during follow-up because of vasculitis flare(s). Thus, they might benefit from a regimen combining glucocorticoids and an immunosuppressant [24].

What is EGPA prognosis? EGPA is now considered a chronic and frequently relapsing disease that requires long-term immunosuppression, unfortunately associated with non-negligible sequelae [25]. Only a minority of patients can be weaned-off glucocorticoids [12] and most require $>7.5 \mathrm{mg}$ of prednisone.day ${ }^{-1}$ (e.g. the EULAR threshold for vasculitis remission), mainly to control their asthma and/or ENT symptoms [26]. Reducing glucocorticoid exposure and preventing treatment-related adverse events are major challenges. Among others, cardiovascular risk factors and osteoporosis should be screened and adequately managed [27], and vaccination against pneumococci and influenza encouraged. The recently published CORTAGE trial showed that AAV patients aged $>65$ years, with or without poor prognosis factors, can benefit from lower immunosuppressant doses, which are associated with less frequent drug-related side-effects [28]. 
What lies ahead? The task force also advanced a future research agenda. First, unlike other AAVs, no EGPA genome-wide association study or animal model is available. Also, unfortunately, no reliable EGPA biomarker has been identified. One can envisage that a good diagnostic biomarker would help physicians differentiate EGPA from eosinophilic asthma and HES, and identify patients with late-onset asthma who are prone to developing vasculitis and, hence, require close monitoring. During follow-up, the total eosinophil count is, at present, the only useful biomarker available to help assess disease activity [29]. Notably, no consensus diagnostic criteria have yet been established. Most studies use classification criteria (e.g. American College of Rheumatology and/or Chapel Hill Nomenclature for Vasculitides) for diagnostic purposes $[1,30]$ but, in the absence of vasculitis (clinical or histological), it may be difficult to distinguish between EGPA and other eosinophilic disorders. The task force members strived during their meetings to define EGPA diagnosis criteria but the debate is not yet closed. Lastly, whether patients might benefit from therapeutic strategies tailored to their ANCA status (e.g. B-cell targeted therapy versus anti-eosinophilic drugs for ANCA-positive and -negative patients, respectively) warrants further investigation.

The EGPA Consensus Task Force's publication is the first attempt by an international and interdisciplinary panel of experts to provide physicians with consensual guidance for the diagnosis and management of EGPA patients. Some recommendations have low evidence levels (and/or are opinion based) and should not be considered definitive. First and foremost, they aim to give physicians tools for individualised EGPA patient management and serve as a starting point for future studies. Fortunately, times are changing and prospective randomised controlled trials, like MIRRA and REOVAS (evaluating mepolizumab and rituximab, respectively), have been designed specifically for EGPA. Like the ongoing revision of the 2009 EULAR recommendations for AAV management, regular EGPA Task Force updates will undoubtedly be necessary to maintain accuracy.

\section{Acknowledgements}

Members of the EGPA Task Force and co-authors of the article cited in this editorial are: Chiara Baldini (Rheumatology Unit, Dept of Internal Medicine, University of Pisa, Pisa, Italy); Elisabeth Bel (Dept of Respiratory Medicine, Academic Medical Centre, University of Amsterdam, Amsterdam, the Netherlands); Paolo Bottero (Allergy and Clinical Immunology Outpatient Clinic, Ospedale "G. Fornaroli" di Magenta, Azienda Ospedaliera di Legnano, Milan, Italy); Jean-François Cordier (Dept of Respiratory Medicine, National Referral Center for Rare Lung Diseases, Hôpital LouisPradel, Hospices Civils de Lyon, Lyon, France); Vincent Cottin (Dept of Respiratory Medicine, National Referral Center for Rare Lung Diseases, Hôpital Louis-Pradel, Hospices Civils de Lyon, Lyon, France); Klaus Dalhoff (Medical Clinic, Dept of Rheumatology, Vasculitis Center, University Clinic of Schleswig-Holstein, Lübeck and Bad Bramstedt, Germany); Bertrand Dunogué (Dept of Internal Medicine, National Referral Center for Rare Autoimmune and Systemic Diseases (Vasculitis, Scleroderma), INSERM U1016, Hôpital Cochin, APHP, Université Paris Descartes, Paris, France); Wolfgang Gross (Medical Clinic, Dept of Rheumatology, Vasculitis Center, University Clinic of Schleswig-Holstein, Lübeck and Bad Bramstedt, Germany); Julia Holle (Medical Clinic, Dept of Rheumatology, Vasculitis Center, University Clinic of Schleswig-Holstein, Lübeck and Bad Bramstedt, Germany); Marc Humbert (Dept of Respiratory and Critical Care Medicine, National Referral Center for Severe Pulmonary Hypertension, INSERM UMR-S 999, Hôpital Bicêtre, APHP, Université Paris-Sud, Le Kremlin-Bicêtre, France); David Jayne (Vasculitis and Lupus Clinic, Addenbrooke's Hospital, Cambridge, UK); J. Charles Jennette (Dept of Pathology and Laboratory Medicine and UNC Kidney Center, University of North Carolina, Chapel Hill, NC, USA); Romain Lazor (Interstitial and Rare Lung Disease Unit, Dept of Respiratory Medicine, Centre Hospitalier Universitaire Vaudois, Lausanne, Switzerland); Alfred Mahr (Dept of Internal Medicine, Hôpital Saint-Louis, Université Paris 7 René Diderot, Paris, France); Peter A. Merkel (Division of Rheumatology, University of Pennsylvania, Philadelphia, PA, USA); Luc Mouthon (Dept of Internal Medicine, National Referral Center for Rare Autoimmune and Systemic Diseases (Vasculitis, Scleroderma), INSERM U1016, Hôpital Cochin, APHP, Université Paris Descartes, Paris, France); Renato Alberto Sinico (Clinical Immunology Unit and Renal Unit, Dept of Medicine, Azienda Ospedaliera San Carlo Borromeo, Milan, Italy); Ulrich Specks (Division of Pulmonary and Critical Care Medicine, Mayo Clinic College of Medicine, Rochester, Minnesota, USA); Augusto Vaglio (Nephrology Unit, University Hospital of Parma, Parma, Italy); and Michael E. Wechsler (Division of Pulmonary, Critical Care and Sleep Medicine, National Jewish Health, Denver, CO, USA).

\section{References}

1 Jennette JC, Falk RJ, Bacon PA, et al. 2012 revised International Chapel Hill Consensus Conference nomenclature of vasculitides. Arthritis Rheum 2013; 65: 1-11.

2 Comarmond C, Pagnoux C, Khellaf M, et al. Eosinophilic granulomatosis with polyangiitis (Churg-Strauss syndrome): clinical characteristics and long-term followup of the 383 patients enrolled in the French Vasculitis Study Group cohort. Arthritis Rheum 2013; 65: 270-281.

3 Keogh KA, Specks U. Churg-Strauss syndrome: clinical presentation, antineutrophil cytoplasmic antibodies, and leukotriene receptor antagonists. Am J Med 2003; 115: 284-290.

4 Valent P, Klion AD, Horny HP, et al. Contemporary consensus proposal on criteria and classification of eosinophilic disorders and related syndromes. J Allergy Clin Immunol 2012; 130: 607-612.

5 Flossmann O, Berden A, de Groot K, et al. Long-term patient survival in ANCA-associated vasculitis. Ann Rheum Dis 2011; 70: 488-494.

6 Mukhtyar C, Guillevin L, Cid MC, et al. EULAR recommendations for the management of primary small and medium vessel vasculitis. Ann Rheum Dis 2009; 68: 310-317.

7 Mahr A, Guillevin L, Poissonnet M, et al. Prevalences of polyarteritis nodosa, microscopic polyangiitis, Wegener's granulomatosis, and Churg-Strauss syndrome in a French urban multiethnic population in 2000: a capturerecapture estimate. Arthritis Rheum 2004; 51: 92-99. 
Groh M, Pagnoux C, Baldini C, et al. Eosinophilic granulomatosis with polyangiitis (Churg-Strauss) (EGPA) Consensus Task Force recommendations for evaluation and management. Eur J Intern Med 2015 [in press; DOI: 10.1016/j.ejim.2015.04.022]

9 Andrews J, Guyatt G, Oxman AD, et al. GRADE guidelines: 14. Going from evidence to recommendations: the significance and presentation of recommendations. J Clin Epidemiol 2013; 66: 719-725.

10 Moosig F, Bremer JP, Hellmich B, et al. A vasculitis centre based management strategy leads to improved outcome in eosinophilic granulomatosis and polyangiitis (Churg-Strauss, EGPA): monocentric experiences in 150 patients. Ann Rheum Dis 2013; 6: 1011-1017.

11 Guillevin L, Lhote F, Gayraud M, et al. Prognostic factors in polyarteritis nodosa and Churg-Strauss syndrome. A prospective study in 342 patients. Medicine (Baltimore) 1996; 75: 17-28.

12 Samson M, Puéchal X, Devilliers $\mathrm{H}$, et al. Long-term outcomes of 118 patients with eosinophilic granulomatosis with polyangiitis (Churg-Strauss syndrome) enrolled in two prospective trials. J Autoimmun 2013; 43: 60-69.

13 Pagnoux C, Mahr A, Hamidou MA, et al. Azathioprine or methotrexate maintenance for ANCA-associated vasculitis. $N$ Engl J Med 2008; 359: 2790-2803.

14 Hiemstra TF, Walsh M, Mahr A, et al. Mycophenolate mofetil vs azathioprine for remission maintenance in antineutrophil cytoplasmic antibody-associated vasculitis: a randomized controlled trial. JAMA 2010; 304: 2381-2388.

15 Jayne D, Rasmussen N, Andrassy K, et al. A randomized trial of maintenance therapy for vasculitis associated with antineutrophil cytoplasmic autoantibodies. N Engl J Med 2003; 349: 36-44.

16 Giavina-Bianchi P, Giavina-Bianchi M, Agondi R, et al. Omalizumab and Churg-Strauss syndrome. J Allergy Clin Immunol 2008; 122: 217.

17 Stone JH, Merkel PA, Spiera R, et al. Rituximab versus cyclophosphamide for ANCA-associated vasculitis. $N$ Engl J Med 2010; 363: 221-232.

18 Guillevin L, Pagnoux C, Karras A, et al. Rituximab versus azathioprine for maintenance in ANCA-associated vasculitis. N Engl J Med 2014; 371: 1771-1780.

19 Mohammad AJ, Hot A, Arndt F, et al. Rituximab for the treatment of eosinophilic granulomatosis with polyangiitis (Churg-Strauss). Ann Rheum Dis 2014 [in press DOI; 10.1136/annrheumdis-2014-206095].

20 Bel EH, Wenzel SE, Thompson PJ, et al. Oral glucocorticoid-sparing effect of mepolizumab in eosinophilic asthma. N Engl J Med 2014; 371: 1189-1197.

21 Moosig F, Gross WL, Herrmann K, et al. Targeting interleukin-5 in refractory and relapsing Churg-Strauss syndrome. Ann Intern Med 2011; 155: 341-343.

22 Kim S, Marigowda G, Oren E, et al. Mepolizumab as a steroid-sparing treatment option in patients with ChurgStrauss syndrome. J Allergy Clin Immunol 2010; 125: 1336-1343.

23 Groh M, Masciocco G, Kirchner E, et al. Heart transplantation in patients with eosinophilic granulomatosis with polyangiitis (Churg-Strauss syndrome). J Heart Lung Transplant 2014; 33: 842-850.

24 Samson M, Puéchal X, Devilliers H, et al. Mononeuritis multiplex predicts the need for immunosuppressive or immunomodulatory drugs for EGPA, PAN and MPA patients without poor-prognosis factors. Autoimmun Rev 2014; 13: 945-953.

25 Robson J, Doll H, Suppiah R, et al. Damage in the ANCA-associated vasculitides: long-term data from the European Vasculitis Study group (EUVAS) therapeutic trials. Ann Rheum Dis 2015; 74: 177-184

26 Hellmich B, Flossmann O, Gross WL, et al. EULAR recommendations for conducting clinical studies and/or clinical trials in systemic vasculitis: focus on anti-neutrophil cytoplasm antibody-associated vasculitis. Ann Rheum Dis 2007; 66: 605-617.

27 Duru N, van der Goes MC, Jacobs JWG, et al. EULAR evidence-based and consensus-based recommendations on the management of medium to high-dose glucocorticoid therapy in rheumatic diseases. Ann Rheum Dis 2013; 72 : 1905-1913

28 Pagnoux C, Quéméneur T, Ninet J, et al. Treatment of systemic necrotizing vasculitides in patients aged sixty-five years or older: results of a multicenter, open-label, randomized controlled trial of corticosteroid and cyclophosphamide-based induction therapy. Arthritis Rheum 2015; 67: 1117-1127.

29 Grayson PC, Monach PA, Pagnoux C, et al. Value of commonly measured laboratory tests as biomarkers of disease activity and predictors of relapse in eosinophilic granulomatosis with polyangiitis. Rheumatology 2015; 54: 1351-1359.

30 Masi AT, Hunder GG, Lie JT, et al. The American College of Rheumatology 1990 criteria for the classification of Churg-Strauss syndrome (allergic granulomatosis and angiitis). Arthritis Rheum 1990; 33: 1094-1100. 